Eco matemático ISSN 1794-8321 E-ISSN 2462-8794 Volumen 8 (s1) 2017, páginas 33 - 37

\title{
Evaluación Contextualizada Como Estrategia Docente Para Potenciar el Desarrollo de Competencias Matemáticas en Pruebas Saber
}

\section{Contextualized evaluation as a teaching strategy to improve the development of mathematic competences in state examination}

\author{
Avaliação contextualizada como estratégia de ensino para melhorar o desenvolvimento de \\ competências matemáticas no exame do estado \\ Sandro Javier Velásquez- Luna, Jorge Leonardo Celis- Gutiérrez, César Augusto Hernández-Suárez ${ }^{\mathrm{c} *}$ \\ a Magister en Innovaciones educativas, Colegio Santo Angel de la Guarda, Cúcuta, Colombia.(D) \\ ${ }^{b}$ Magister Neuro Psicología, Colegio Santo Angel de la Guarda Cúcuta, Colombia. (D) \\ Mắgister en las Enseñanza Ciencias Básicas mención Matemáticas, Universidad Francisco de Paula Santander, Cúcuta, Colombia.
}

\begin{abstract}
Forma de citar: Velásquez, S. Celis, J. Hernández, C. (2016). Evaluación Contextualizada Como Estrategia Docente Para Potenciar el Desarrollo de Competencias Matemáticas en Pruebas Saber. Encuentro Internacional en Educación Matematica ISSN 2539-1885. La Educación Matematica como Herramienta en el Desempeño Profesional Docente. Cúcuta, Colombia. 33 - 37.
\end{abstract}

\begin{abstract}
Resumen: La presente investigación plantea la evaluación contextualizada como estrategia docente para potenciar el desarrollo de competencias matemáticas en pruebas saber. Pretende diagnosticar estrategias de mejoramiento, determinar factibilidad y proponer procesos evaluativos para desarrollar competencias matemáticas según los lineamientos de la prueba saber. La investigación se realiza con la finalidad de que el docente logre fomentar y desarrollar en sus estudiantes competencias para el razonamiento cuantitativo, Interpretación y representación, Formulación y ejecución y Razonamiento y argumentación, mediante contextos dados en la nueva alineación del sistema nacional de evaluación estandarizada. Para el logro del mismo fue necesario observar la realidad existente, por lo tanto; la presente investigación se ubica dentro del paradigma cuantitativo de tipo proyecto factible, su diseño de investigación es no experimental de nivel descriptivo y su modalidad es de campo. El análisis realizado ha permitido observar resultados y comparativos de las pruebas saber 11 en diferentes instituciones de educación básica y media, como también la realidad de procesos evaluativos implementados por los docentes, los cuales necesitan formación continua en procesos de evaluación. Es necesario un cambio en la forma de evaluar y procesos de acción en cuanto a estrategias de mejoramiento se refiere. En este sentido, la evaluación contextualizada se plantea como una respuesta al empeño por mejorar los índices de calidad y cambiar la manera de evaluar, de igual manera intenta tomar la evaluación para transformarla en un proceso formativo que sea mucho más significativo y aplique estrategias de mejoramiento con acompañamiento docente.
\end{abstract}

Palabras clave Competencias, Contexto, Evaluación, Pruebas saber

\begin{abstract}
The following investigation set up the contextualized evaluation as a teaching strategy to improve the development of mathematic competences in the state examination. It intends to diagnose the improvement strategies, determine the viability and propose evaluating processes to develop the mathematic competences according to the guidelines of the state examination the investigation was made with the aim that the teacher could encourage and develop in his/her students quantitative reasoning competences, interpretation and representation, formulation and execution and reasoning and argumentation. Through given contexts in the new standardize national system line up. In order to achieve this it was necessary to observe the reality, there for, the following investigation is placed inside the quantitative paradigm of a feasibility study type, its investigation design is non experimental of a descriptive level and its method is a field method. The preformed analysis has allowed us to observe comparative results of the state examination of $11^{\circ}$ in different institutions of primary and high school education. Since also the reality of the evaluating process implemented for teachers, who need constant preparation in evaluating processes. It is necessary a change in the way of evaluate and action processes improvement strategies related. In that sense, the contextualized evaluation is set up as an answer to the will of improve the quality rates and change the way of evaluate, at the same time trying to take evaluation in order to transform it in a formative process which would be much more meaningful and apply improvement strategies with the teacher's accompaniment.
\end{abstract}

Keywords Competences, Context, Evaluation, State examination

* Autor para correspondencia cesaraugusto@ufps.edu.co

2590-9215@ 2017 Universidad Francisco de Paula Santander. Este es un artículo bajo la licencia CCBY 
Resumo: A investigação a seguir configurou a avaliação contextualizada como uma estratégia de ensino para melhorar o desenvolvimento de competências matemáticas no exame do estado. Ela pretende diagnosticar as estratégias de melhoria, determinar a viabilidade e propor avaliação dos processos para desenvolver as competências matemáticas de acordo com as diretrizes do exame do estado a investigação foi feita com o objectivo de que o professor pode estimular e desenvolver em seus / suas alunos competências de raciocínio quantitativo, interpretação e representação, formulação e execução e raciocínio e argumentação. Em todos os contextos da nova linha de padronização do sistema nacional. Para alcançar isso, era necessário observar a realidade, e por isso, a seguinte investigação é colocado dentro do paradigma quantitativo de um tipo de estudo de viabilidade, o seu design é a investigação não experimental de um nível descritivo e seu método é um campo método. A análise pré-formada nos permitiu observar resultados comparativos do exame do $11^{\circ}$ estado em diferentes instituições de ensino fundamental e médio. Desde também a realidade do processo de avaliação implementado para os professores, que precisam de constante preparação na avaliação de processos. É necessário mudar o modo de ação e processar as estratégias de melhoria relacionadas. Nesse sentido, a avaliação contextualizada é configurá-lo como uma resposta à vontade de melhorar os índices de qualidade e mudar a maneira de evaluate, ao mesmo tempo tentando tirar de avaliação, a fim de transformá-lo em um processo formativo que seria muito mais significativas e aplicar estratégias de melhoria com o acompanhamento do professor.

\section{Palavras chave Competências, Contexto, Avaliação, Exame do Estado}

\section{Introducción}

El conocimiento y las situaciones de aprendizaje de las matemáticas se presentan como un pilar que sostienen las vivencias del ser humano, la globalización y la nueva era del conocimiento en todas las áreas muestran que el proceso de enseñanza necesita cada vez mas de estrategias que permitan fomentar los aprendizajes necesarios para aplicarlos en situaciones de la vida diaria y estimular el deseo de aprender por sí mismo.

Por lo tanto, la educación matemática exige un nuevo enfoque fundamentado en un buen proceso evaluativo que permita incorporar el desarrollo de competencias desde contextos adecuados a situaciones interesantes a cada estudiante. Con respecto a la evaluación contextualizada Arredondo (2010) Afirma que "la Practica Evaluadora, no es solo desarrollo de la instrucción o transmisión de conocimientos, sino, sobre todo, la información intelectual en contenidos y estrategias cognitivas, el logro de competencias y la educación en valores y actitudes de los alumnos como estudiantes y como ciudadanos de nuestra sociedad".

Es así como la evaluación plantea la aplicación de las matemáticas en contextos reales, donde el docente evalúa para formar. Luego, el propósito principal de la evaluación es beneficiar al estudiante de una cultura matemática que le permita comprender y solucionar situaciones reales de la mejor manera posible en su entorno personal, social y profesional.

Así mismo, Tobón (2008) define las competencias como: "procesos complejos de desempeño con idoneidad en determinados contextos, integrando diferentes saberes (saber ser, saber hacer, saber conocer y saber convivir), para realizar actividades y/o resolver problemas con sentido de reto, motivación, flexibilidad, creatividad, comprensión y emprendimiento".
Lo que explica que las competencias se basan en la aplicación de diferentes saberes en contextos variados y ambientes de aprendizaje enriquecidos por situaciones problema, que propician el avance de niveles de competencias cada vez con mayor nivel de complejidad. Es decir, las competencias se manifiestan en un conjunto de habilidades que posee el ser humano para facilitar el desempeño y aplicarlo en actividades contextualizadas a situaciones individuales. En este sentido Yepes (2010), explica que "la evaluación puede servir como un motivador para el desempeño de los estudiantes. Si estos aprenden a valorar la evaluación como indicador de desempeño, se esforzaran por lograr cada vez un mejor rendimiento". Este autor afirma que la evaluación es útil siempre y cuando exista retroalimentación entre maestros, estudiantes y padres de familia, luego así puede ser un gran factor de motivación entre todos los desempeños de alto nivel y obtener un rendimiento adecuado.

Sin embargo, contrasta la poca atención que recibe la evaluación formativa en el aula con la importancia que se le ha dado a los exámenes estandarizados, puesto que los resultados en las competencias matemáticas obtenidas en la prueba del (programa internacional de evaluación de estudiantes).

PISA realizadas en los últimos años no son satisfactorios, ya que, Educativa, R. (2008) "en el año 2006 el menor desempeño se registró en matemáticas, donde la quinta parte (18\%) de los evaluados alcanzo el nivel mínimo", la mayoría de los estudiantes colombianos solo demostró la capacidad para identificar información y llevar a cabo procedimientos matemáticos y responder a preguntas relacionadas con contextos conocidos. Para Núñez (2002) La baja calidad de la educación en Colombia es preocupante. Las pruebas SABER, realizadas por el ministerio de Educación Nacional, revelaron que en secundaria una cuarta parte de los estudiantes de colegios públicos no logro superar el nivel mínimo de las pruebas de matemáticas y menos del $20 \%$ alcanzo el nivel óptimo ICFES (2013). 
Además los resultados sobre las pruebas del año 2012 por parte del Instituto Colombiano para el Fomento de la educación Superior ICFES (2013), indican que, al igual que los demás países latinoamericanos participantes, tienen desempeños inferiores al promedio de los países de (La Organización para la Cooperación y el desarrollo Económico) OCDE.

Por lo que Leyva, J \& otros (2006). "Solo dos de cada Diez estudiantes pueden hacer interpretaciones literales de los resultados de problemas matemáticos". Aunque estos resultados en las pruebas saber que evalúan competencias matemáticas no son favorables para los esfuerzos que viene realizando el gobierno nacional.

Así mismo, El Ministerio de Educación Nacional de Colombia (Plan Sectorial 2006 2010) señala que uno de los indicadores más importantes del nivel de calidad de la educación en el país es, sin lugar a dudas, el desempeño de los estudiantes en pruebas que miden el nivel de desarrollo de competencias.

Es decir, que las pruebas saber indican en gran parte la calidad de los procesos de enseñanza aprendizaje en cada una de las instituciones del país, puesto que los resultados están enfocados en la evaluación por competencias. Al mismo tiempo, se observó la ausencia de procesos formativos dentro de la evaluación y falta de aplicación de problemas para desarrollar niveles de competencias matemáticas para motivar el aprendizaje en los jóvenes de instituciones educativas de la ciudad de Cúcuta.

Esta situación afecta notablemente los resultados, avances y metas de calidad propuestas en las diferentes instituciones educativas y por consiguiente en la entidad territorial, consecuencia de la falta de preparación, investigación y actualización docente en cuanto a estrategias de evaluación se refiere.

Todo lo anterior, evidencia que se debe propiciar ambientes de aprendizaje dentro de procesos de evaluación formativa y contextualizada que permitan implementar estrategias que promuevan el desarrollo de competencias para potenciar la motivación del estudiante y por ende buen rendimiento académico.

En concordancia con lo anterior Bustinza (2006) afirma "lograr elevados niveles de aprendizaje supone saber que cada estudiante aprende según sus propias motivaciones, su nivel de desarrollo cognitivo-académico y su propio estilo de aprendizaje". Lo que explica que se requiere que el estudiante se apropie de su proceso cognitivo y que el docente evalué según su ritmo de aprendizaje, analicen juntos situaciones contextualizadas con las matemáticas en los diferentes niveles de competencias y logren alcanzar los estándares de competencia propuestos por el Ministerio de Educación Nacional (MEN).
Además, el decreto 1290 de 2009 propuesto por el MEN explica que son propósitos de la evaluación institucional de los estudiantes "identificar las características personales, intereses, ritmos de desarrollo y estilos de aprendizaje del estudiante para valorar sus avances". Expuestos los argumentos teóricos se diagnosticaron estrategias empleadas por docentes de diferentes instituciones de la ciudad de Cúcuta y se realizó la propuesta atendiendo su factibilidad.

\section{Materiales y métodos}

La presente investigación se ubica dentro del paradigma cuantitativo de tipo proyecto factible, su diseño de investigación es no experimental de nivel descriptivo y su modalidad es de campo.

Sujetos Objeto de Estudio. Esta investigación se realizó con docentes de matemáticas de diferentes instituciones educativas de la ciudad de Cúcuta, del sector público y del privado que asisten a los encuentros de la Red de Experiencias Matemáticas de Norte de Santander, anualmente en el colegio Santo Ángel de la Guarda Cúcuta. La Población está conformada por los docentes de educación básica y media que trabajan en el área de matemáticas en la secretaría de educación de San José de Cúcuta. La muestra está conformada por 25 docentes para conocer las opiniones con respecto a la evaluación y estrategias de mejoramiento que ellos conocen y utilizan con los estudiantes de básica y media académica en cuanto al desarrollo de competencias matemáticas y pruebas saber. Además para este estudio se tuvo en cuenta los promedios de matemáticas en pruebas saber de 2015 de la entidad territorial de la ciudad de Cúcuta y la media Nacional.

\section{Etapas}

Fase I Diagnostica En esta fase se elaboró una encuesta (escala de Likert con respuestas de selección)que fue aplicada a los docentes para conocer las opiniones con respecto a los procesos de evaluación y estrategias de mejoramiento que ellos conocen y utilizan con respecto a los resultados que obtienen los estudiantes en las pruebas saber en matemáticas. Esto, permitió comprobar la necesidad de diseñar una propuesta: Evaluación Contextualizada Para Potenciar el Desarrollo de Competencias Matemáticas en Pruebas Saber, con el objeto de aumentar los índices de calidad de las instituciones educativas de la región.

\section{Fase II Diseño}

Dentro del diseño se consideraron los siguientes aspectos:

Componente Disciplinar: Se seleccionaron las competencias correspondientes a cada nivel educativo de acuerdo a los estándares, derechos básicos de aprendizaje y matrices de referencia propuestas por el gobierno nacional para el mejoramiento de las pruebas saber en matemáticas. 
Sandro Javier Velásquez- Luna, Jorge Leonardo Celis- Gutiérrez, César Augusto Hernández-Suárez

\begin{tabular}{ccc}
\hline \multicolumn{3}{c}{ Tabla I. Intervalos de comparación } \\
\hline Rango de Actuación & Categorías & Criterios \\
\hline $1-1,99$ & Nunca $(N)$ & Deficiente \\
$2-2,99$ & Casi Nunca $(C N)$ & Regular \\
$3-3,99$ & Algunas Veces (AV) & Bueno \\
$4-4,99$ & Casi Siempre (CS) & Muy Bueno \\
5 & Siempre (S) & Excelente \\
\hline \multicolumn{3}{c}{ Fuente: Velásquez (2016) }
\end{tabular}

Componente Pedagógico: Recursos de diseño curricular en relación a las actividades para la enseñanza, Procesos de evaluación formativa, rubrica, el aprendizaje y la evaluación, el uso del portafolio como evidencia de avances del proceso.

Aspectos a Tener en Cuenta en La Aplicación de la Estrategia: Para aplicar la propuesta que corresponde a la aplicación de la estrategia Evaluación Contextualizada Para Potenciar el Desarrollo de Competencias Matemáticas en Pruebas Saber es importante tener en cuenta que el docente debe conocer detalladamente los estudiantes y sus ritmos de aprendizaje, estudiar las competencias básicas propuestas para cada estándar, diseñar procesos de evaluación diagnostica y evaluación formativa.

De acuerdo a ello se debe tener en cuenta diseñar las evaluaciones atendiendo a los contextos propuestos por el ICFES en el sistema nacional de evaluación estandarizada: Contextos de matemáticas Financieras, contextos de divulgación científica, contextos sociales y contextos ocupacionales.

Asimismo el diseño de preguntas que promuevan la práctica de las competencias matemáticas: Interpretación y representación, Formulación y ejecución y Razonamiento $\mathrm{y}$ argumentación. Además se propone aplicar los formatos de resultados esperados en el plan de mejoramiento propuesto

Finalmente hay que concientizar al estudiante de la importancia que tiene su papel activo en la evaluación mediante la rúbrica y el portafolio de evidencias.

\section{Resultados y discusión}

La información obtenida hace parte de un estudio de estadística descriptiva, es decir se tabularon los datos obtenidos y se aplicaron fórmulas estadísticas para obtener comparaciones teniendo en cuenta el instrumento de recolección de datos.

Además se tuvo en cuenta el siguiente baremo para calcular los resultados (Tabla I). Se analizaron aspectos teóricos y metodológicos para sustentar la propuesta. A continuación los resultados de acuerdo a cada uno de los objetivos y las variables en estudio

Evaluación Contextualizada Los resultados obtenidos muestran que los docentes conocen diferentes tipos de evaluación, implementan en su praxis docente procedimientos diagnósticos, y de evaluación Sumativa pero no están preparados para aplicar un proceso de evaluación formativa, puesto que esta variable tiene una media de 2,7 con criterio regular. En relación a la definición de contextualización y práctica de una evaluación que potencie el desarrollo de competencias matemáticas este indicador muestra poco avance a pesar de los esfuerzos que se hacen por mejorar los resultados de las pruebas en algunas instituciones, teniendo en cuenta que se obtuvo una media de 2,88 que corresponde al criterio regular.

Competencias Matemáticas. Para el dominio de Leyva (2006) Competencia en matemáticas concierne a la capacidad de los estudiantes para analizar, razonar y comunicar eficazmente a sus ideas al tiempo que se plantean, formulan, resuelven e interpretan tareas matemáticas en una variedad de contextos.

Al respecto, los resultados muestran que los docentes en cuanto concepto de competencias en general, Obtuvieron una media aritmética de 3.88 con relación a su definición, lo que quiere decir que es un criterio bueno pero de sebe profundizar aún más. Es decir, que casi siempre existen docentes con conocimientos pertinentes en los aspectos teóricos que tienen que ver con competencias. De igual manera, los datos logrados en cuanto a competencias como saber hacer en un contexto corresponden al criterio regular, puesto que competencia como habilidades se representa con una media de 2,9 y el indicador estándares de matemáticas con una media de 3,46 corresponde a un criterio bueno según el análisis estadístico operado.

Pruebas Saber. Con respecto a los conocimientos que tienen los docentes sobre la prueba saber y el análisis de resultados en sus respectivas instituciones se obtuvo una media de 3,5 ubicado en el criterio bueno.

Sin embargo con respecto al desarrollo de planes de mejoramiento funcionales y aplicados con seguimiento adecuado los docentes manifestaron poca credibilidad y poca funcionalidad a estos procesos con una media de 1,7 que es deficiente, es decir, que para los docentes el seguimiento a resultados a la prueba saber con planes de mejoramiento no es un elemento primordial para mejorar procesos de enseñanza aprendizaje en cuanto a potenciar las competencias matemáticas.

En cuanto a la factibilidad de ejecutar una propuesta de Evaluación Contextualizada Para Potenciar el Desarrollo de Competencias Matemáticas en Pruebas Saber los docentes señalan que los colegios cuentan con herramientas técnicas, legales y políticas para llevar a cabo las mismas, ya que alcanzó un promedio de 4,2 con criterio Muy bueno, eso quiere decir; que se cuenta con lo necesario para desarrollar actividades implementando la propuesta, luego existe factibilidad educativa, los profesores alcanzaron a señalar que es factible realizar pedagógicamente la propuesta con una media de 4,4. 
Conclusiones y Recomendaciones Se verificó con base en los resultados mostrados en las tablas estadísticas y gráficos, que para aplicar la propuesta es importante tener en cuenta que la evaluación contextual debe estar inmersa en el proceso educativo y transformarse en instrumento de acción pedagógica que permita, adaptar el proceso de enseñanza aprendizaje a las características individuales de los alumnos.

De igual manera el docente debe conocer detalladamente los estudiantes y sus ritmos de aprendizaje, estudiar las competencias básicas propuestas para cada estándar, diseñar procesos de evaluación diagnostica y evaluación formativa De acuerdo a ello, el docente debe tener en cuenta diseñar las evaluaciones atendiendo a los contextos propuestos por el ICFES en el sistema nacional de evaluación estandarizada: Contextos de matemáticas Financieras, contextos de divulgación científica, contextos sociales y contextos ocupacionales. Asimismo el diseño de preguntas que promuevan la práctica de las competencias matemáticas: Interpretación y representación, Formulación y ejecución y Razonamiento y argumentación. Además se propone aplicar los formatos de resultados esperados en el plan de mejoramiento propuesto. Finalmente hay que concientizar al estudiante de la importancia que tiene su papel activo en la evaluación mediante la rúbrica y el portafolio de evidencias.

Con respecto a los resultados obtenidos en la aplicación del instrumento, se concluye que existe factibilidad institucional, educativa y pedagógica para implementar la propuesta. Se concluye que se requiere el planteamiento de una propuesta que permita integrar la evaluación contextual en el proceso educativo como instrumento de acción pedagógica según las características individuales de los alumnos. Asimismo, el docente requiere preparación y actualización en cuanto a la contextualización de las matemáticas enfocadas a la prueba saber y la ejecución de planes de mejoramiento y seguimiento a los resultados.

Para finalizar, se propone, establecerlos desempeños, indicadores, evidencias, capacidades y habilidades pueden activar los alumnos en la solución de problemas, es decir, evaluar hasta qué puntos son matemáticamente competentes para desarrollar problemas en situaciones reales, para el diseño de una evaluación formativa y real.

\section{Referencias}

Arredondo, S. C., Diago, J.C., \& Cañizal, A. (2010). Evaluación educativade aprendizajes y competencias. Pearson Prentice Hall.

Arredondo, S. C., Diago, J.C., \& Cañizal, A. (2010). Evaluación educativade aprendizajes y competencias. Pearson Prentice Hall.
Bustinza, C., Durán, D. y Quintasi, J. (2006). Diagnóstico de estilos de aprendizaje de estudiantes del IV Ciclo de la especialidad de educación inicial. Resultados de investigación educativa. Recuperado el 12 de octubre de 2008, de http:// ww.tarea.org.pe/modulos/pdf/ClaudiaBustinza_Diagnostico Estilos.pdf.

Decreto 1290. Por el cual se reglamenta la evaluación del aprendizaje y promoción de los estudiantes de los niveles de educación básica y media.

Educativa, R. (2008).PlanSectorial 2006-2010. Ministerio de Educación Nacional. Disponible en: http://www.mineducacion.gov.co/1621/articles-152025_recurso_1_pdf.pdf.

ICFES (2013,Diciembre).Colombiaen PISA 2012. [Datos en Línea]. En ICFES: Informe nacional de resultados Resumen ejecutivo. Bogotá, D.C. Disponible: http://repository.udistrital.edu.co/bitstream/11349/2304/2/Be 1tranCast roArietaC ecilia2015.JPG.pdf. Consulta: 2015, Noviembre 03.

Leyva, J. L. L., \& Garrido, Y. P. (2006).

Reflexiones sobre la calidad del aprendizaje y de las competencias matemáticas. Revista Iberoamericana de Educación, 41(1), 3. Ministerio de Educación Nacional (2009).

Núñez, J. C., González-Pienda,J.A., Alvarez, L., González-Castro, P., González- Pumariega, S., Roces, C., \& Da Silva, E.H. (2005). Las actitudes hacia las matemáticas: perspectiva evolutiva. In Actas do VIII Congreso Galaico-Portugués de Psicopedagoxía (pp.2389-2396).

Nuñez, J et all., 2002"¿Qué pasa con los Colegios públicos?”, Cuartillas de Economía $\mathrm{N}^{\circ}$, pp. 2-3.

Sarmiento G. V. (2008).Colombia: qué y cómo mejorar a partir de la prueba PISA. [Documento en Línea] Periódico Al tablero No. 44, enero-marzo 2008 del Ministerio de Educación Nacional. Disponible: http://www.mineducacion.gov.co/1621/propert yvalue-37909.html [Consulta:2015, Noviembre 03

Tobón, S. (2008). La formación basada en competencias en la educación superior: el enfoque complejo. México: Universidad Autónoma de Guadalajara.

Yepes, R. L. G. (2010). Calidad Educativa: Más que resulta dos en pruebas estandarizadas. Revista Educación y Pedagogía, 16(38). 